\title{
Learning the changes of barnase mutants thermostability from structural fluctuations obtained using anisotropic network modeling
}

\author{
Nikolay Alemasov \\ Kurchatov Genomics Center, \\ Institute of Cytology and Genetics \\ SB RAS \\ Novosibirsk, Russia \\ ORCID iD: 0000-0002-7511-5385
}

\author{
Nikita Ivanisenko \\ Kurchatov Genomics Center, \\ Institute of Cytology and Genetics \\ SB RAS \\ Novosibirsk, Russia \\ ivanisenko@bionet.nsc.ru
}

\author{
Vladimir Ivanisenko \\ Kurchatov Genomics Center, \\ Institute of Cytology and Genetics \\ SB RAS \\ Novosibirsk, Russia \\ salix@bionet.nsc.ru
}

\begin{abstract}
In biotechnology applications, rational design of new proteins with improved physico-chemical properties includes a number of important tasks. One of the greatest practical and fundamental challenges is the design of highly thermostable protein enzymes that maintain catalytic activity at high temperatures. This problem may be solved by introducing mutations into the wild-type enzyme protein. In this work, to predict the impact of such mutations in barnase protein we applied the anisotropic network modeling approach, revealing atomic fluctuations in structural regions that are changed in mutants compared to the wild-type protein. A regression model was constructed based on these structural features that can allow one to predict the thermal stability of new barnase mutants. Moreover, the analysis of regression model provides a mechanistic explanation of how the structural features can contribute to the thermal stability of barnase mutants.
\end{abstract}

Keywords - barnase, thermostability, free energy, prediction, machine learning

\section{Introduction}

The thermal stability of proteins reflects the ability to preserve the unique spatial structure of the polypeptide chain under high temperature. Thermostability is determined numerically through the differences between the enthalpy and entropy of the protein in the native and denatured states [1] and can be determined from the thermodynamic properties of the amino acids that make up the protein [2]. There are several experimental methods for improving the thermal stability of a protein: stabilizing its native form, destabilizing its denatured form, or by combining both approaches [3].

The present work is aimed at developing an approach to construct protein structure "profiles" indicating the positions of amino acid residues that are most sensitive in terms of protein thermal stability to mutations. Such approaches were previously developed to study the survival time of patients with mutations in the SOD1 protein. Here, the feasibility of this approach to assess thermal stability was shown for the first time using barnase protein as an example [4].

\section{Methods}

The experimental thermal stability of 134 barnase mutants as assessed by Gibbs free energy ( $\left.\mathrm{ddG}, \mathrm{kJmol}^{-1}\right)$ was taken from the work of Seeliger and de Groot [5].

The initial structure for the study was taken from the PDB (1BNI). Mutant structures were obtained by introducing an appropriate mutation using the PDBFixer tool (https://github. com/pandegroup/pdbfixer). The dynamics of the barnase mutants and their wild types were simulated using anisotropic network models (ANM) implemented in the ProDy [6]. The simulation was performed with the following parameters: $m \in$ $\{16,48,80,159\}, n=10, A \in\{0.5,1,2,3\}$ A, where ${ }^{\circ} m$ is the number of first vibrational modes, $n$ is the number of conformations obtained, $A$ is the desired root-meansquared deviation from the original structure. Each of the $n$ conformations obtained for a given barnase mutant was subjected to geometry optimization using the RDKit package (http://www.rdkit.org/).

To obtain the properties of the protein structure, after the simulation the MDTraj was used [7]. The following measures were calculated using MDTraj for each conformation: secondary structure (DSSP), gyration radius $(\mathrm{Rg})$, root-meansquared fluctuations of protein residues (RMSF), and solvent accessible surface area (SASA) of each residue. The random forest method implemented using the scikit-learn was used to construct the regression models [8]. Stability selection was used as a feature selection algorithm [9].

The training set included $90 \%$ of the mutants while the test set contained $10 \%$ of the mutants. At each bootstrap step for the mutants from the test sample, the rootmeansquared prediction error (RMSE) of the Gibbs free energy was estimated.

The treeinterpreter (https:/github.com/andosa/ treeinterpreter) method of interpreting the results of predictions obtained using the random forest was used to study the mechanism influencing the mutations that affect the thermal stability of barnase.

\section{Results}

As a result of the ANM simulation of the wild-type protein, 318 modes with non-zero eigenvalues were obtained. In total, 136 regression models were constructed (on 16 single parameters and 120 various pairs of simulation parameters) using the random forest method, linking the calculated parameters of the structure of the barnase mutants and the thermal stability of these mutants.

The most accurate regression model was a model based on a pair of variants $\left(A=0.5 \mathrm{~A},{ }^{\circ} m=80\right.$ and $A=2 \mathrm{~A},{ }^{\circ} m=$ 159) with the accuracies RMSE $=5.06 \pm 1.27 \mathrm{kJmol}^{-1}$ and AUE $=3.87 \pm 0.06 \mathrm{kJmol}^{-1}$. Hereinafter, this model will be denoted by its serial number 114 among all 136 models. 
TABLE I - TOP TEN IMPORTANT FOR PREDICTING THE THERMAL STABILITY OF BARNASE MUTANTS USING REGRESSION MODEL NO. 114 FEATURES. THE NAME OF THE FEATURE CONTAINS A CALCULATED PARAMETER (DSSP, RMSF OR SASA), AN INDICATION OF THE AVERAGE VALUE OF THE PARAMETER (BAR) OR STANDARD DEVIATION FROM THE MEAN $(\Sigma)$, AS WELL AS THE NUMBER OF AMINO ACID RESIDUES IN THE SEQUENCE INDICATED AFTER "R"

\begin{tabular}{|c|c|c|c|c|}
\hline$\#$ & $\mathrm{~A}, \mathrm{~A}^{\circ}$ & $\mathrm{m}$ & Feature name & $\begin{array}{c}\text { Normalized feature } \\
\text { importance, a.u. }\end{array}$ \\
\hline 1 & 0.5 & 80 & RMSF, r21 & $0.05 \pm 0.02$ \\
2 & 2.0 & 159 & $\overline{ }$ & $0.05 \pm 0.03$ \\
3 & 0.5 & 80 & SASA, r3 & $0.02 \pm 0.02$ \\
& & & $\overline{\text { SASA }, \mathrm{r} 88}$ & \\
4 & 0.5 & 80 & RMSF, r47 & $0.02 \pm 0.01$ \\
5 & 0.5 & 80 & RMSF, r20 & $0.02 \pm 0.02$ \\
6 & 2.0 & 159 & RMSF, r41 & $0.02 \pm 0.01$ \\
7 & 2.0 & 159 & $\sigma$ (SASA), r23 & $0.02 \pm 0.02$ \\
8 & 0.5 & 80 & $\sigma$ (SASA), r74 & $0.02 \pm 0.01$ \\
9 & 2.0 & 159 & RMSF, r34 & $0.02 \pm 0.01$ \\
10 & 0.5 & 80 & & $0.02 \pm 0.01$ \\
& & & SASA, r74 & \\
\hline
\end{tabular}

While constructing the regression model No. 114 using the stable selection method, 29 features important for predictions were selected. The first ten features with the highest importance are presented in table I. Among the top ten important features were those associated with amino acid residues at positions 3, 20-21, 23, 34, 41, 47, 74 and 88. An analysis of the contributions of each of the features selected as important for predicting the thermal stability of the barnase mutants revealed that deviations in the values of five features (\#1, \#2, \#6, \# 8, \# 10, see table I) account for more than $80 \%$ of the variance in the contributions of all features.

\section{Discussion}

Previously, three hydrophobic cores were found in barnase [10]. In the present work, features associated with residues at the positions Val3, Leu20, Pro21, Asn23, Gly34, Asn41, Pro47, Ala74, and Ile88 were found to be among the most important ones for predicting thermostability. Of these amino acid residues, Ile88 is located in the hydrophobic core 1. The residues at positions 20,21 , and 23 are located on the same loop as the Ile25 residue from the core 2, and the Asn41 residue is located in the same alpha helix as Leu42 from core 2. The Ala74 is located in the same beta-strand as the Ile 76 from the hydrophobic core 1 . It can be assumed that the barnase mutants studied here can promote structural changes affecting significant elements, such as hydrophobic core 1 and core 2 .

The computational analysis of the barnase mutants conducted in the current work leads to the following conclusion: it is possible to determine the kind (stabilization or destabilization) of the mutational effect on the structure of the protein based on the remaining three features: the average surface area accessible to the solvent in the region of the amino acid residues Val3 and Ala74; the deviation of this area in the region of the residue Ala74 from the average; the mean-square fluctuations in the region of the residues Pro21 and Asn41 in mutants. Such an opportunity can allow us to propose new mutations in this protein that will stabilize its structure compared to the wild-type protein.

\section{ACKNOWLEDGMENT}

The authors thank the Siberian Branch of the Russian Academy of Sciences for the budget project № 032420190040-C-01 and the Russian Foundation for Basic Research together with the Government of the Novosibirsk Region for the Project № 19-44-543002.

\section{REFERENCES}

[1] S. Talluri, "Advances in engineering of proteins for thermal stability", International Journal of Advanced Biotechnology and Research, vol. 2 (1), pp. 190-200, 2011.

[2] C. Tanford, "Contribution of Hydrophobic Interactions to the Stability of the Globular Conformation of Proteins", Journal of the American Chemical Society, vol. 84 (22), pp. 4240-4247, 1962.

[3] B. W. Matthews, "Structural and genetic analysis of protein stability", Annual review of biochemistry, vol. 62, pp. 139-60, 1993.

[4] N. Alemasov, N. Ivanisenko and V. Ivanisenko, "Learning the changes of barnase mutants thermostability from structural fluctuations obtained using anisotropic network modeling", Journal of Molecular Graphics and Modelling, vol. 97 (107572), pp. 1-9, 2020

[5] D. Seeliger and B. L. de Groot, "Protein thermostability calculations using alchemical free energy simulations", Biophysical journal, vol. 98 (10), pp. 2309-2316, 2010

[6] A. Bakan, L. M. Meireles, and I. Bahar, "ProDy: Protein Dynamics Inferred from Theory and Experiments", in Bioinformatics, vol. 27 (11), pp. 1575-1577, 2011.

[7] R. T. McGibbon et al., "MDTraj: A Modern Open Library for the Analysis of Molecular Dynamics Trajectories", Biophysical Journal, vol. 109 (8), pp. 1528-1532, 2015.

[8] F. Pedregosa et al., "Scikit-learn: Machine Learning in Python", Journal of Machine Learning Research, vol. 12, pp. 2825-2830, 2011.

[9] N. Meinshausen and P. Buhlmann, "Stability selection", Journal of the" Royal Statistical Society. Series B: Statistical Methodology, vol. 72 (4), pp. 417-473, 2010.

[10] A. R. Fersht, "Protein folding and stability: the pathway of folding of barnase", FEBS Letters, vol. 325 (1-2), pp. 5-16, 1993. 